\title{
Experimental and Theoretical Study of 4-Methylaminoantipyrine with Divalent Metal Ions
}

\author{
Farah M. Ibrahim \\ Department of Chemistry, college of Science, Al-Nahrain University, Baghdad-Iraq. \\ Corresponding author: farahtaha2016@gmail.com
}

\begin{abstract}
$\mathrm{Co}^{2+}, \mathrm{Ni}^{2+}, \mathrm{Cu}^{2+}$ complexes with 4-methylaminoantipyrine (MAP) were synthesis and characterized by IR, UV-Vis., thermal analysis, CHNO-S analysis, magnetic susceptibility, conductivity measurements and this work includes a theoretical study of MAP complexes where it was done by the program of hyperchem8.0.7 using semi-empirical calculations. The PM3 method at $298 \mathrm{~K}$ used to calculate geometric properties, binding energy $(\Delta \mathrm{Eb})$, heat of formation $\left(\Delta \mathrm{H}^{\circ} \mathrm{f}\right)$, total energy ( $\Delta$ Etot.), ultraviolet and vibrational data of the MAP complexes. The comparing of experimental data with theoretical data gave good results, so the square planar geometry suggested for complexes. [DOI: $10.22401 / \mathrm{JNUS} .21 .3 .08]$
\end{abstract}

Keywords: 4-methylaminoantipyrine, divalent metal ions, thermal analysis.

\section{Introduction}

Antipyrine, aminopyrine and 4methylaminoantipyrine compounds are used as analgesic, anti-inflammatory, antipyretic and anticancer drugs [1-4]. N-heterocyclic inhibition against pathogenic bacteria and fungi [5]. 4-methylaminoantipyrine and 4aminoantipyrine are strong inhibitors of cyclooxygenase in the treatment of inflammatory pain [6-7]. 4-aminoantipyrine and its derivatives characterized by ${ }^{1} \mathrm{H}-\mathrm{NMR}$, single-crystal X-ray diffraction and FTIR techniques, the theoretical vibration frequencies show good result with the experimental vibration frequencies data [8]. New derivatives of 4-aminoantipyrin have anti-breast cancer activity due to pyrazole, pyrrole and pyrimidine moieties were synthesis and characterized by elemental analysis and ${ }^{13} \mathrm{C},{ }^{1} \mathrm{H}$ NMR, IR spectral [9]. Schiff bases derived from 4-aminophenazone were prepared and the structure of compounds investigated FT-IR, NMR, Mass studies, elemental analysis and screened to be antibacterial active agents[10-12]. 4aminoantipyrine used as an inhibitor for the corrosion of mild steel in $0.5 \mathrm{M}$ sulphuric acid solution, thermodynamics of adsorption were calculated. Quantum chemical calculations was calculate the electronic properties to expect the inhibitive effect of the compound [13]. The aim of work is prepared and characterized complexes of compound has pharmacology applications and compared these data with computational calculation using Hyperchem 8.0.7 program to suggest the accurate structure of MAP complexes that which proposed the square planar geometry finally.

\section{Experimental \\ Instrumentation}

Infrared spectra of (MAP) complexes measured by ALPHA FTIR spectrophotometer. Shimadzu UV-Vis 160A spectrophotometer were used for UV-Visible spectra of (MAP) complexes. Shimadzu 680 cc-flame measured the metal ion percent. CHNS-O analysis was carried out on EURO EA elemental analyzer. Thermal analyses (TGDTG) were gained on a LINSEIS (STA PT1000). Johnson Mattey's magnetic susceptibility balance can be used for paramagnetic and diamagnetic materials. Molar conductivity measurements carried by corning conductivity meter 220. Melting points of ligand and its complexes were measured by Gallenkamp M.F.B. 600.01 apparatus.

\section{Preparation of MAP complexes}

The divalent complexes were prepared by mixing aqueous solution $(10 \mathrm{ml})$ of metal salts $\left(\mathrm{CuSO}_{4} .5 \mathrm{H}_{2} \mathrm{O}, \mathrm{CoCl}_{2} .2 \mathrm{H}_{2} \mathrm{O}\right.$ and $\left.\mathrm{NiSO}_{4} \cdot 6 \mathrm{H}_{2} \mathrm{O}\right)$ with aqueous solution $(10 \mathrm{ml})$ of MAP 1:2 (metal: ligand) mole ratio and refluxed for 12 hours. A colored precipitate was formed at room temperature, filtered and washed with distilled water then dried in oven at $50^{\circ} \mathrm{C}$. 
Table (1)

Some characterization data of MAP and metal complexes.

\begin{tabular}{|c|c|c|c|c|c|c|c|c|}
\hline \multirow{2}{*}{ Compound } & \multirow{2}{*}{$\begin{array}{c}\text { Conductivity, } \\
\text { DMF solvent } \\
\mu \mathrm{s} / \mathrm{cm} \\
\end{array}$} & \multirow{2}{*}{$\begin{array}{l}\text { Melting } \\
\text { Point, }{ }^{\circ} \mathrm{C} \\
\text { colour } \\
\end{array}$} & \multicolumn{6}{|c|}{$\begin{array}{c}\text { Found\% } \\
\text { (Calculate)\% }\end{array}$} \\
\hline & & & $\bar{C}$ & $\mathrm{H}$ & $\mathbf{O}$ & $\mathbf{N}$ & $\mathbf{S}$ & Metal \\
\hline MAP & - & $\begin{array}{c}\text { White } \\
110 \\
\end{array}$ & $\begin{array}{r}66.30 \\
(66.36) \\
\end{array}$ & $\begin{array}{r}6.90 \\
(6.91) \\
\end{array}$ & $\begin{array}{c}7.35 \\
(7.37) \\
\end{array}$ & $\begin{array}{c}19.30 \\
(19.35) \\
\end{array}$ & - & - \\
\hline CoMAP & 146 & $\begin{array}{c}\text { Light pink } \\
119-121 \\
\end{array}$ & $\begin{array}{c}51.00 \\
(51.08) \\
\end{array}$ & $\begin{array}{c}5.30 \\
(5.32) \\
\end{array}$ & $\begin{array}{c}5.60 \\
(5.67) \\
\end{array}$ & $\begin{array}{c}14.80 \\
(14.89) \\
\end{array}$ & - & $\begin{array}{c}10.43 \\
(10.45) \\
\end{array}$ \\
\hline NiMAP & 78 & $\begin{array}{c}\text { Light green } \\
120-122 \\
\end{array}$ & $\begin{array}{c}48.90 \\
(48.92)\end{array}$ & $\begin{array}{c}5.04 \\
(5.09)\end{array}$ & $\begin{array}{c}16.26 \\
(16.31)\end{array}$ & $\begin{array}{l}14.20 \\
(14.27)\end{array}$ & $\begin{array}{l}5.40 \\
(5.43)\end{array}$ & $\begin{array}{c}9.95 \\
(9.97)\end{array}$ \\
\hline CuMAP & 85 & $\begin{array}{c}\text { Light green } \\
122-124 \\
\end{array}$ & $\begin{array}{c}48.50 \\
(48.52) \\
\end{array}$ & $\begin{array}{c}5.00 \\
(5.05) \\
\end{array}$ & $\begin{array}{c}16.12 \\
(16.17) \\
\end{array}$ & $\begin{array}{c}14.10 \\
(14.15) \\
\end{array}$ & $\begin{array}{c}5.30 \\
(5.39) \\
\end{array}$ & $\begin{array}{c}10.68 \\
(10.70) \\
\end{array}$ \\
\hline
\end{tabular}

\section{IR Spectra}

IR of MAP: A stretching vibration band at $\left(3319.77 \mathrm{~cm}^{-1}\right),\left(1652.34 \mathrm{~cm}^{-1}\right),\left(3108.6 \mathrm{~cm}^{-}\right.$ $\left.{ }^{1}\right),\left(1610.09-1560.53 \mathrm{~cm}^{-1}\right),\left(2877.09 \mathrm{~cm}^{-1}\right)$, (2707.86 $\left.\mathrm{cm}^{-1}\right),\left(2793.51 \quad \mathrm{~cm}^{-1}\right), \quad$ that corresponds to $(\mathrm{NH}),(\mathrm{C}=\mathrm{O}),(\mathrm{CH}$ aromatic $)$, $(\mathrm{C}=\mathrm{C}$ aromatic $), \quad(\mathrm{CH}$ asymmetric $), \quad(\mathrm{CH}$ asymmetric), ( $\mathrm{CH}$ symmetric) groups, respectively and bending vibration band at $\left(1367.93 \mathrm{~cm}^{-1}\right),\left(1435.68 \mathrm{~cm}^{-1}\right),\left(1505.33 \mathrm{~cm}^{-1}\right)$ that corresponds to $(\mathrm{CH}$ symmetric $),(\mathrm{CH}$ asymmetric), $(\mathrm{NH})$ groups, respectively $[8,14]$. Coordination of the $\pi$ electrons $\mathrm{C}=\mathrm{O}$ reduces the double bond character of the $\mathrm{C}-\mathrm{O}$ bond causing absorption at lower wave number, also the donor amino group shift due to the complexation with metal ion, see Fig.(1).

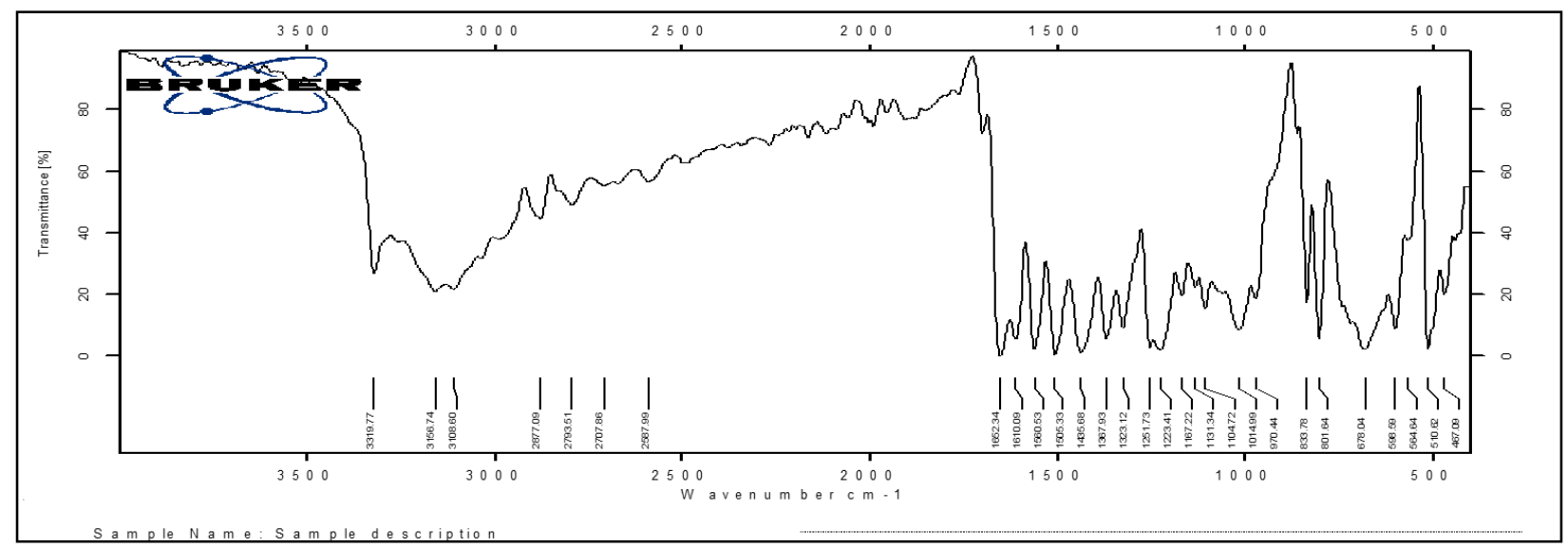

Fig.(1): IR spectrum of MAP.

Experimental IR of CoMAP: A stretching vibration band at $\left(3332.75 \mathrm{~cm}^{-1}\right),\left(1634.65 \mathrm{~cm}^{-1}\right)$ that corresponds to $(\mathrm{NH}),(\mathrm{C}=\mathrm{O})$ groups, respectively [15], see Fig.(2) and Table (2).

Table(2)

Experimental and Theoretical stretching vibration band for $\operatorname{CoMAP}\left(\mathrm{cm}^{-1}\right)$.

\begin{tabular}{||l|l|c|c||c||}
\hline \multicolumn{1}{|c|}{ Compound } & \multicolumn{3}{|c||}{ Frequency } & Intensity \\
\hline \hline \multirow{3}{*}{ CoMAP } & & Theoretical & Experimental & \\
\cline { 2 - 5 } & NH (amine group) & 3329 & 3332.75 & 100.00 \\
\cline { 2 - 5 } & C=O & 1639 & 1634.65 & 80.28 \\
\cline { 2 - 5 } & C-H ( aromatic ring) & 3003 & 3010 & 81.88 \\
\cline { 2 - 5 } & C-H (aliphatic) & 2900 & 2897.6 & 81.36 \\
\cline { 2 - 5 } & C=C( aromatic ring) & 1512 & 1510.34 & 163.13 \\
\hline
\end{tabular}




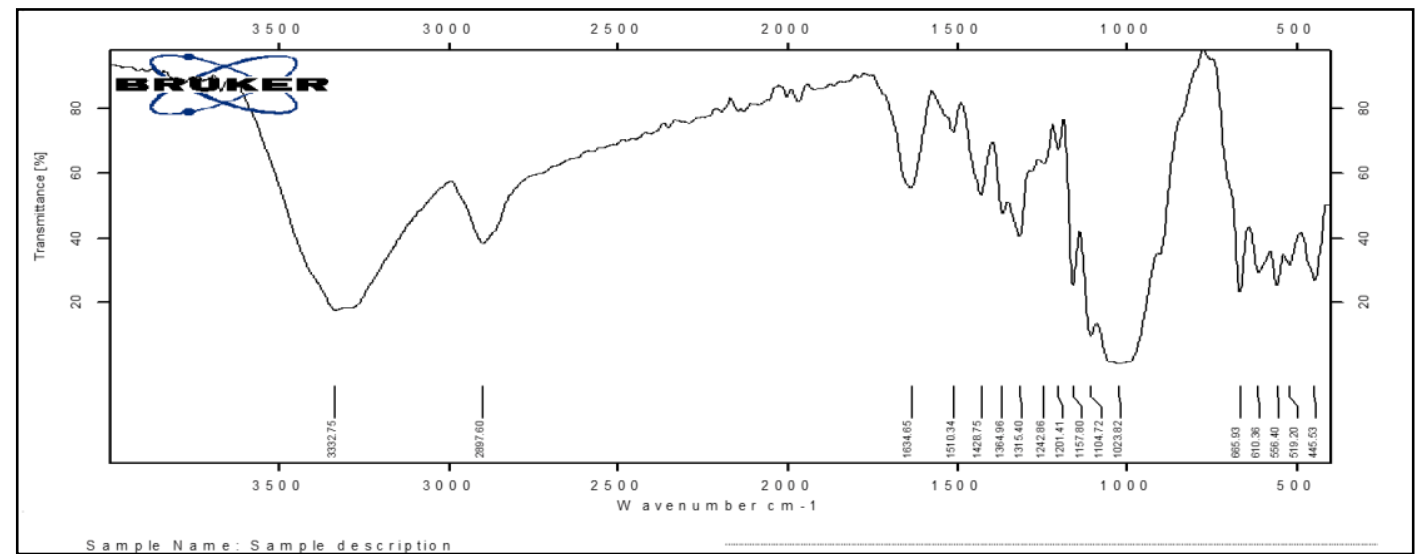

Fig.(2): IR spectrum of CoMAP.

Experimental IR of NiMAP compound: A stretching vibration band at $\left(3331.06 \mathrm{~cm}^{-1}\right)$, $\left(1646.98 \mathrm{~cm}^{-1}\right)$ that corresponds to $(\mathrm{NH})$, $(\mathrm{C}=\mathrm{O})$ groups stretching vibrations, respectively [15], see Fig.(3) and Table (3).

\section{Table(3)}

Experimental and Theoretical stretching vibration band for $\operatorname{NiMAP}\left(\mathrm{cm}^{-1}\right)$.

\begin{tabular}{||l|l|c|c|c||}
\hline Compound & \multicolumn{3}{|l|}{ Frequency } & Intensity \\
\hline \hline \multirow{4}{*}{ NiMAP } & & Theoretical & experimental & \\
\cline { 2 - 5 } & NH (amine group) & 3340 & 3331.06 & 88.00 \\
\cline { 2 - 5 } & C=O & 1648 & 1646.98 & 81.32 \\
\cline { 2 - 5 } & C-H (aromatic ring) & 3003 & 3000 & 79.88 \\
\cline { 2 - 5 } & & & 2942.52 & \\
& C-H (aliphatic) & 2895 & 2890.09 & 82.31 \\
& & & 2890.09 & \\
\cline { 2 - 5 } & C=C( aromatic ring) & 1512 & 1513.56 & 149.22 \\
\hline
\end{tabular}

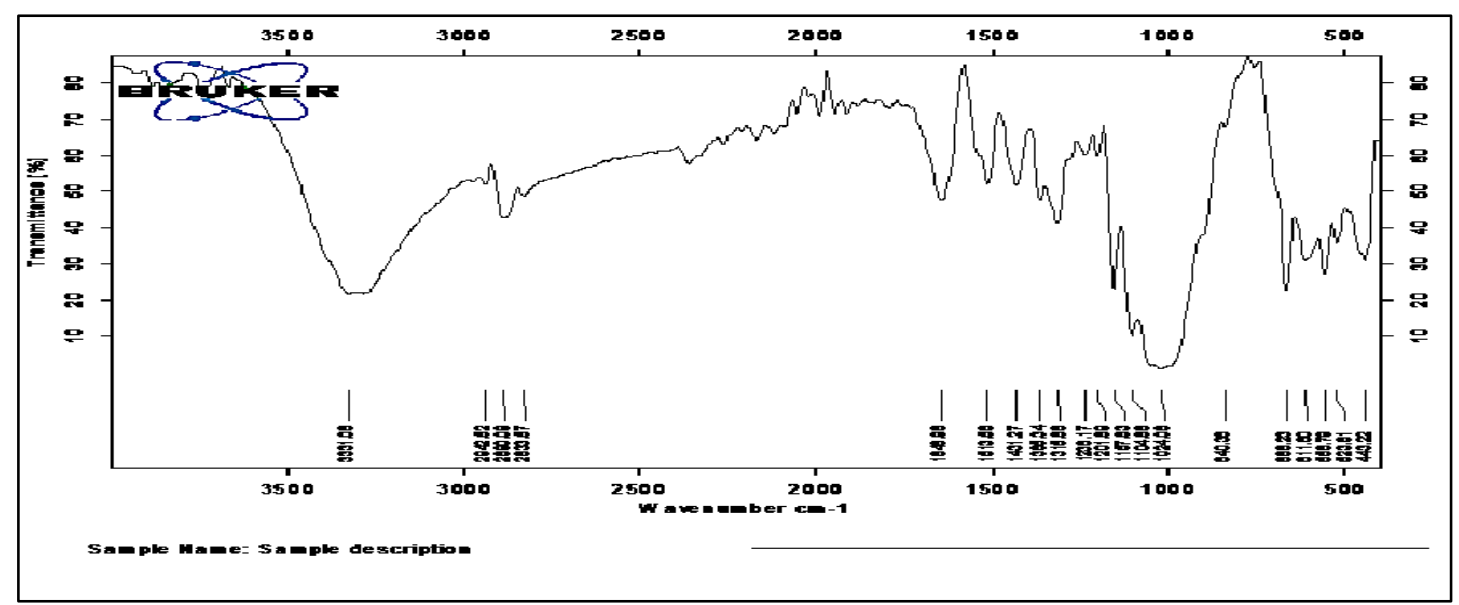

Fig.(3):IR spectrum of NiMAP.

Experimental IR of CuMAP compound: A stretching vibration band at $\left(3282.23 \mathrm{~cm}^{-1}\right)$, $\left(1640.79 \mathrm{~cm}^{-1}\right)$ that corresponds to $(\mathrm{NH})$, $(\mathrm{C}=\mathrm{O})$ groups, respectively [16], see Fig.(4) and Table (4). 
Table(4)

Experimental and Theoretical stretching vibration band for $\operatorname{CuMAP}\left(\mathrm{cm}^{-1}\right)$.

\begin{tabular}{||l||l||c|c||c||}
\hline \multicolumn{1}{|l|}{ Compound } & \multicolumn{3}{|c|}{ Frequency } & Intensity \\
\hline \hline \multirow{6}{*}{ CuMAP } & & Theoretical & experimental & \\
\cline { 2 - 5 } & NH (amine group) & 3268 & 3282.23 & 88.00 \\
\cline { 2 - 5 } & $\mathrm{C}=\mathrm{O}$ & 1645 & 1640.79 & 76.24 \\
\cline { 2 - 5 } & C-H (aromatic ring) & 3003 & 3000.0 & 83.82 \\
\cline { 2 - 5 } & C-H (aliphatic) & 2877 & $\begin{array}{c}289589 \\
2959.88\end{array}$ & 80.30 \\
\cline { 2 - 5 } & C=C(aromatic ring) & 1508 & $1550.99-1502.0$ & 89.47 \\
\hline
\end{tabular}

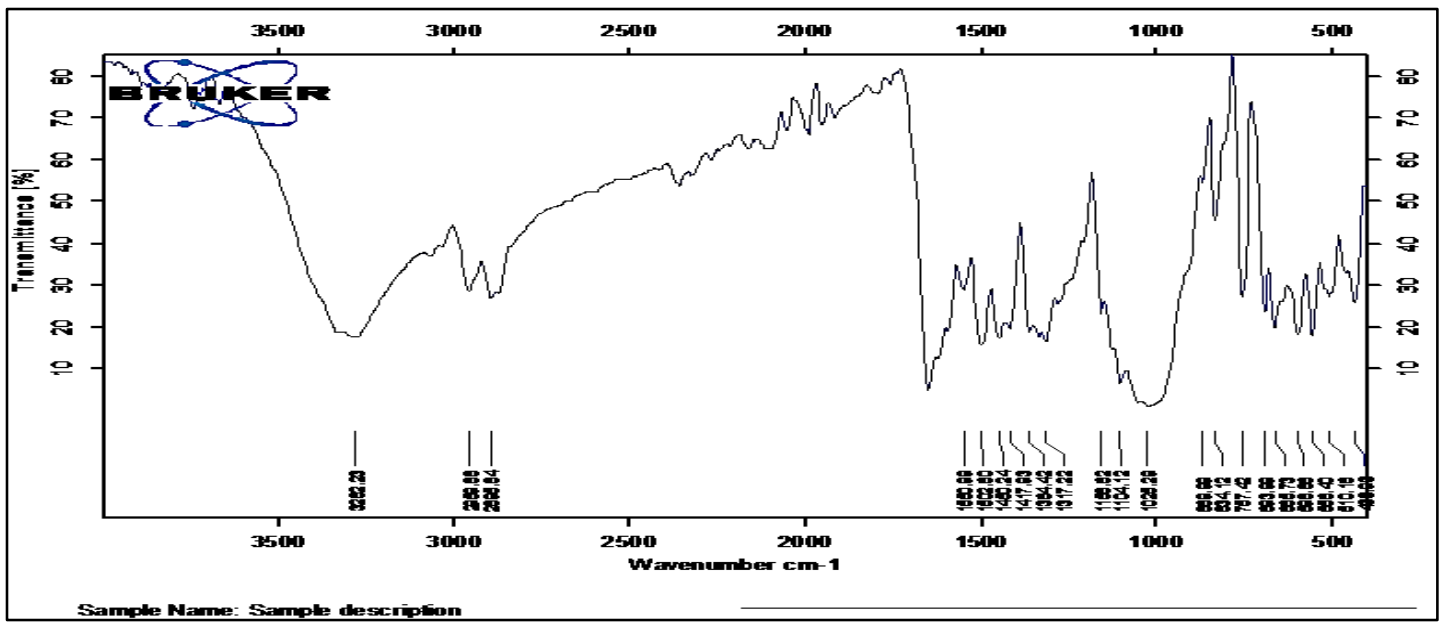

Fig.(4): IR spectrum of CuMAP.

Ultraviolet-Visible spectroscopy and magnetic susceptibility

MAP spectrum showed bands at $(216,234$, 266) $\mathrm{nm}$ were for $\pi \rightarrow \pi^{*}$ electronic transition and at (305) nm for $\mathrm{n} \rightarrow \pi^{*}$ transition. Co MAP complex showed a band at $511 \mathrm{~nm}$ which is assigned to $\left({ }^{1} \mathrm{~A}_{1} \mathrm{~g} \rightarrow{ }^{1} \mathrm{~B}_{1} \mathrm{~g}\right)$ transition. NiMAP complex have bands at $630 \mathrm{~nm}$ assigned to $\left({ }^{1} \mathrm{~A}_{1} \mathrm{~g} \rightarrow{ }^{1} \mathrm{~B}_{1} \mathrm{~g}\right)$ transition, CuMAP showed band at $559 \mathrm{~nm}$ assigned to ${ }^{2} \mathrm{~B}_{1} \mathrm{~g} \rightarrow{ }^{2} \mathrm{~A}_{1} \mathrm{~g}$ transition, so square planar geometry were suggested for $\mathrm{Co}^{2+}, \mathrm{Ni}^{2+}$ and $\mathrm{Cu}^{2+}$ complexes [12].

Table(5)

Comparison of experimental and theoretical ultraviolet for complexes and $\mu$ eff.

\begin{tabular}{|c||c|c||c||c||}
\hline \multicolumn{1}{|c||}{ Compound } & \multicolumn{2}{|c|}{ Experimental } & Theoretical & $\mu$ eff.B.M. \\
\cline { 2 - 5 } CoMAP & $\pi \rightarrow \pi^{*}(\mathrm{~nm})$ & 240.0 & 245 & 2.5 \\
\hline \hline & $\mathrm{n} \rightarrow \pi^{*}(\mathrm{~nm})$ & $342.0,397$ & 321 & - \\
\hline \multirow{2}{*}{ NiMAP } & $\pi \rightarrow \pi^{*}(\mathrm{~nm})$ & 236 & 272 & zero \\
\hline \multirow{2}{*}{ CuMAP } & $\mathrm{n} \rightarrow \pi^{*}(\mathrm{~nm})$ & 330 & 310 & - \\
\hline \hline & $\pi \rightarrow \pi^{*}(\mathrm{~nm})$ & 260 & 257 & 1.94 \\
\hline & $\mathrm{n} \rightarrow \pi^{*}(\mathrm{~nm})$ & 281 & 277 & - \\
\hline
\end{tabular}




\section{Thermal analysis of metal complexes}

The thermo-gravimetry analysis help to investigated the structure of complexes, first loss of $\left[\mathrm{CoC}_{24} \mathrm{H}_{30} \mathrm{~N}_{6} \mathrm{O}_{2} \mathrm{Cl}_{2}\right]$ gave $\left[\mathrm{CoC}_{4} \mathrm{H}_{2} \mathrm{~N}_{6}\right]$ at $279-340 \mathrm{C}$, the other loss at $380-500^{\circ} \mathrm{C}$ for elimination $\left(\mathrm{N}_{4} \mathrm{C}_{4} \mathrm{H}_{2}\right)$ and remain [CoN2]. $\left[\mathrm{NiC}_{24} \mathrm{H}_{30} \mathrm{~N}_{6} \mathrm{O}_{6} \mathrm{~S}\right]$ complex decomposes between $130-320^{\circ} \mathrm{C}$ to form $\left[\mathrm{NiC}_{2} \mathrm{~N}_{4} \mathrm{O}_{6} \mathrm{~S}\right]$ due to the elimination part $\left(\mathrm{C}_{22} \mathrm{H}_{30}\right)$ and the second loss is $\left(\mathrm{N}_{4} \mathrm{C}_{2} \mathrm{O}_{3} \mathrm{~S}\right)$ between $390-560{ }^{\circ} \mathrm{C}$ to residue [NiO],[17].[ $\left[\mathrm{CuC}_{24} \mathrm{H}_{30} \mathrm{~N}_{6} \mathrm{O}_{6} \mathrm{~S}\right]$ complex is assigned to the elimination of $\left(\mathrm{C}_{18} \mathrm{H}_{22}\right.$ $\mathrm{O}_{2} \mathrm{~N}_{4}$ ), the other loss of $\left[\mathrm{CuC}_{6} \mathrm{H}_{8} \mathrm{~N}_{2} \mathrm{O}_{4} \mathrm{~S}\right]$ is $\left(\mathrm{C}_{4} \mathrm{H}_{8} \mathrm{~N}_{4} \mathrm{O}_{3} \mathrm{~S}\right)$ between $330-490{ }^{\circ} \mathrm{C}$ to remain $[\mathrm{CuO}]$.

\section{Theoretical Study}

Program hyperchem-8.0.7 was used for calculations of the heat of formation $\left(\Delta \mathrm{Hf}^{\mathrm{o}}\right)$, and binding energy $(\Delta \mathrm{Eb})$ for MAP complexes were calculated by the semi-empirical and molecular mechanics Table (6). Also, PM3 was used to evaluate the vibrational spectra of MAP complexes. It has been found that these obtained frequencies agree well with the experimental results Table (2-4), HOMO, LUMO and electrostatic potential as shown in Fig.(8), Bond length and bond angle measurements for the MAP complexes was calculated Tables (7-12) Optimization geometry of MAP complexes Figures (5-7) was obtained the PM3 method.

\section{$\operatorname{Table}(6)$}

Conformation energetic (in KJ. mol ${ }^{-1}$ ), HOMO and LUMO energetic Dipole moment.

\begin{tabular}{|c|c|c|c||c|c|c||}
\hline $\begin{array}{c}\text { Compd. } \\
\text { No. }\end{array}$ & $\begin{array}{c}\boldsymbol{\Delta} \mathbf{E}_{\text {tot }} \\
\mathbf{k J} / \mathbf{m o l}\end{array}$ & $\begin{array}{c}\Delta \mathbf{H}_{\mathbf{f}}^{\mathbf{0}} \\
\mathbf{k J} / \mathbf{m o l}\end{array}$ & $\begin{array}{c}\Delta \mathbf{E}_{\mathbf{b}} \\
\mathbf{k J} / \mathbf{m o l}\end{array}$ & $\mathbf{E}_{\text {HOMo }} \mathbf{e V}$ & $\mathbf{E}_{\mathbf{L U M O}} \mathbf{e V}$ & $\begin{array}{c}\text { Dipole moment } \\
\text { (Debye) }\end{array}$ \\
\hline \hline CoMAP & -611750.464 & -1255.660 & -29615.774 & -8.147772 & -2.0694 & 3.4965 \\
\hline NiMAP & -697298.57824 & -2880.4329 & -25859.295 & -6.07491 & -1.80383 & 10.2390 \\
\hline CuMAP & -722855.705 & -1103.278 & -30840.556 & -8.523952 & -0.3874507 & 8.7554 \\
\hline
\end{tabular}

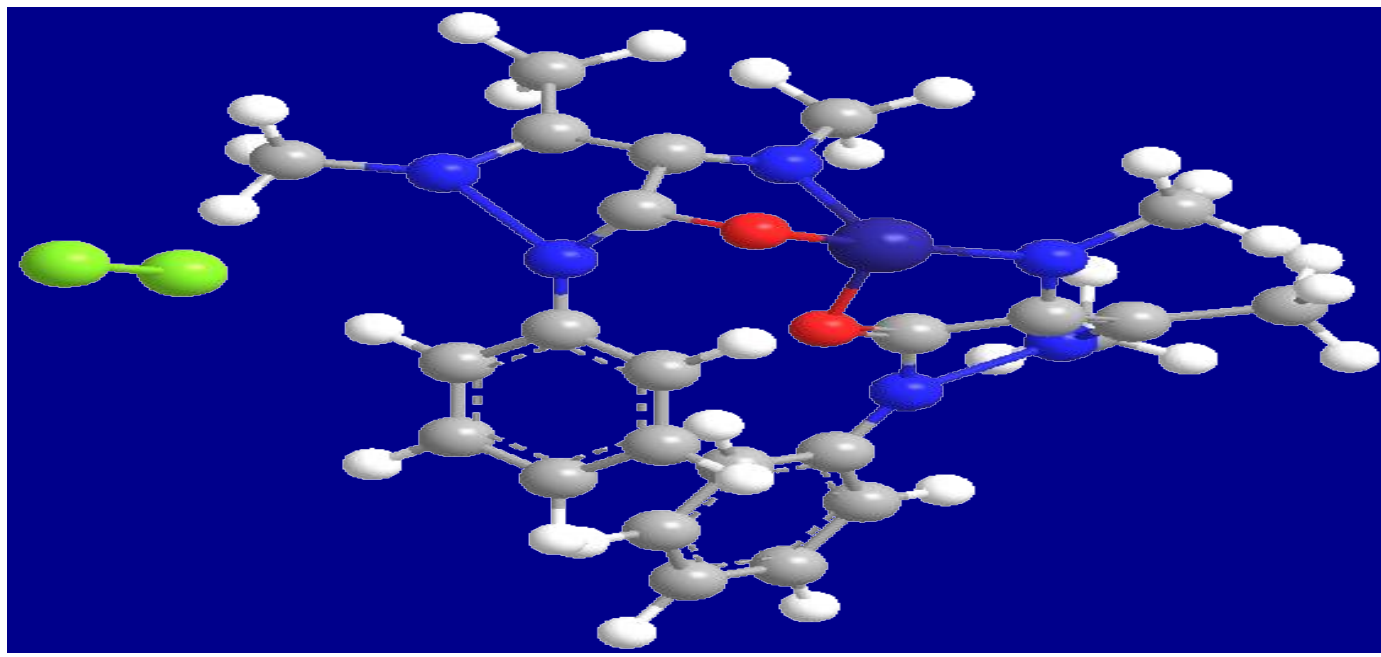

Fig.(5): Optimized geometric for CoMAP.

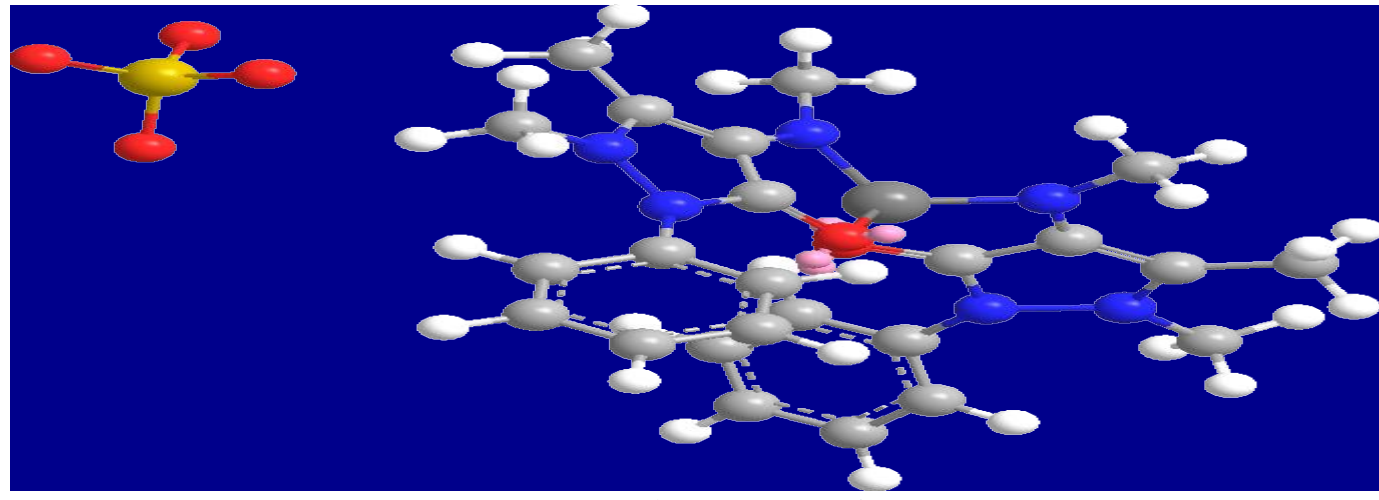

Fig.(6): Optimized geometric for NiMAP. 


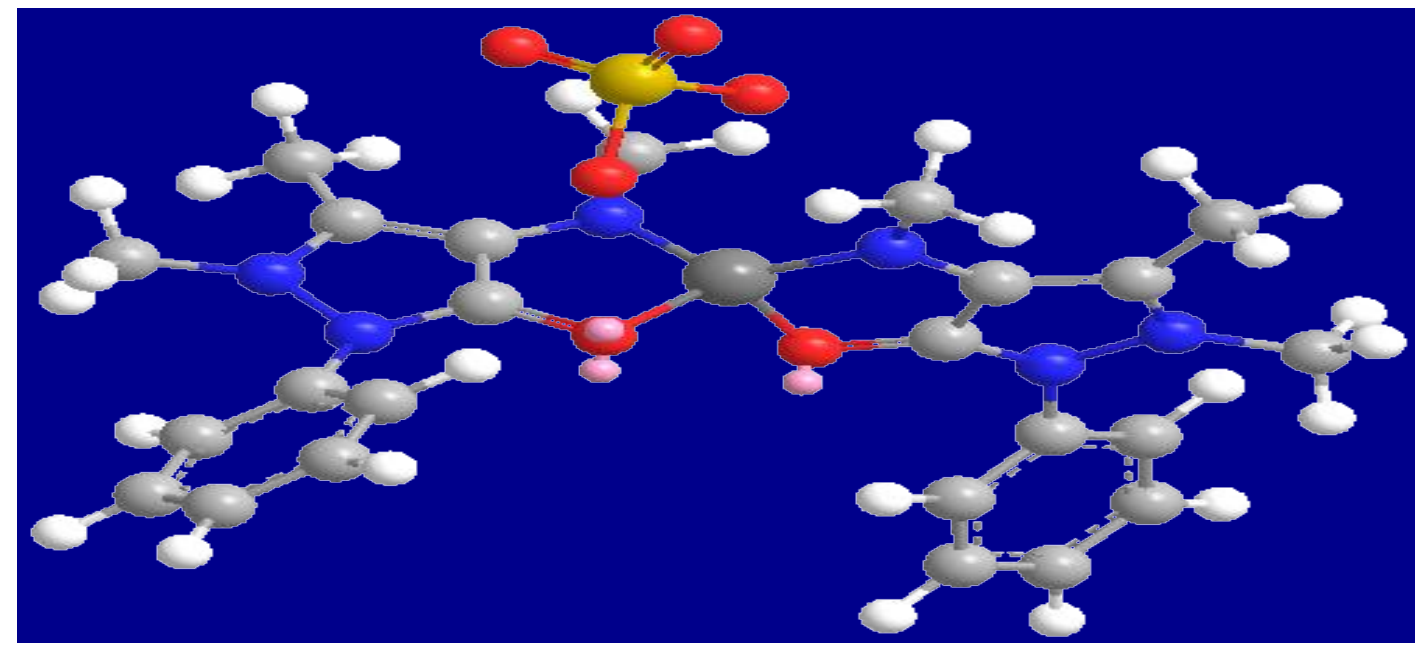

Fig.(7): Optimized geometric for CuMAP.

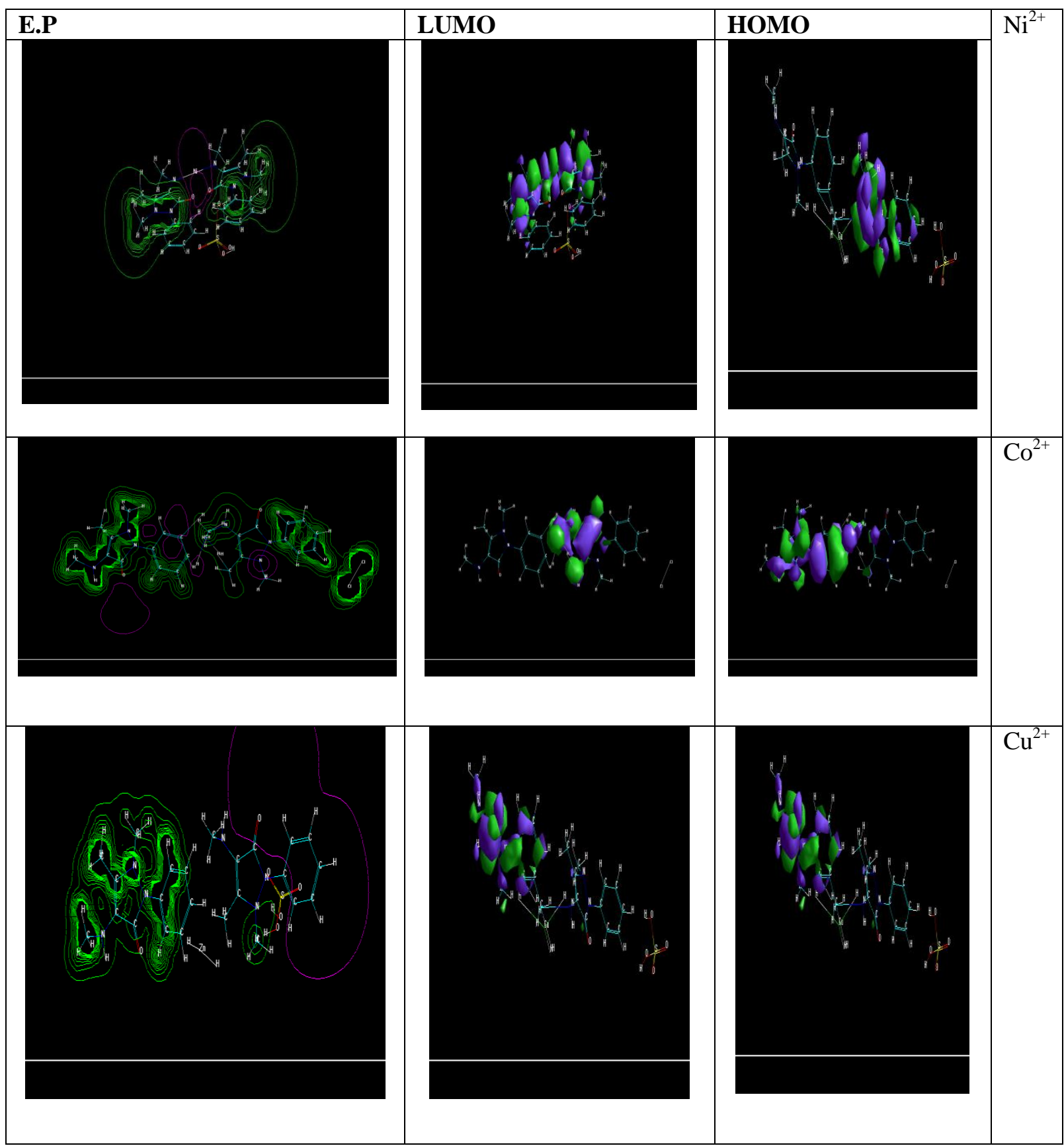

Fig.(8): Electrostatic potential, HOMO and LUMO for complexes. 

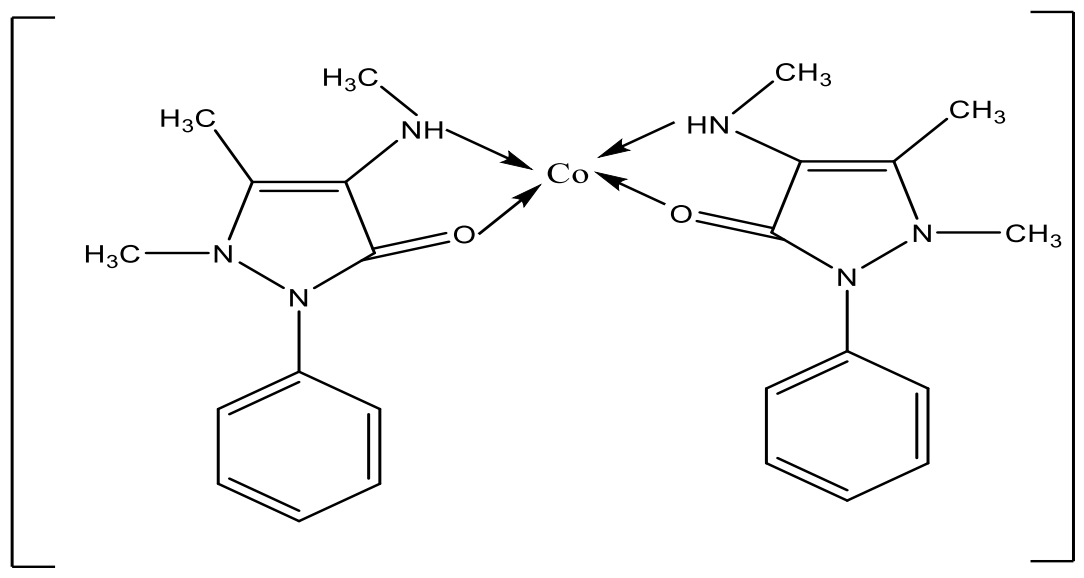

$\mathrm{Cl}_{2}$

Chemical Formula: $\mathrm{C}_{24} \mathrm{H}_{30} \mathrm{Cl}_{2} \mathrm{CoN}_{6} \mathrm{O}_{2}$ Exact Mass: 563.11

Molecular Weight: 564.37

$\mathrm{m} / \mathrm{z}: 563.11(100.0 \%), 565.11(64.5 \%), 564.12(26.4 \%), 566.11(18.0 \%), 567.11$ $(10.6 \%), 565.12(3.8 \%), 568.11(3.0 \%), 567.12(2.4 \%), 564.11(2.2 \%)$

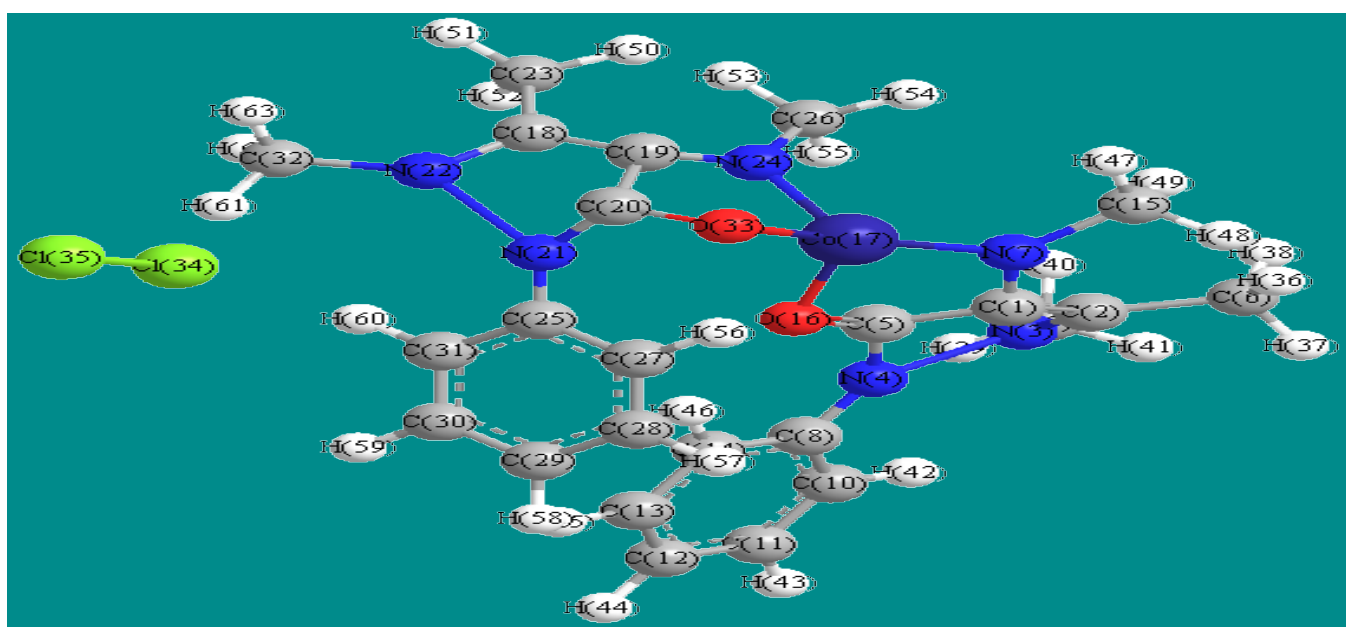

Fig.(9): Serial number of atoms of CoMAP.

Table(7)

Selected molecular structure parameters of Bond lengths $(\stackrel{\AA}{)}$ of CoMAP.

\begin{tabular}{|c|c|c|c|c|c|c|c|c|}
\hline Atoms & Actual & optinal & Atoms & Actual & gotimal & Atpoms & Actalal & gitimal \\
\hline $\begin{array}{c}c(37- \\
H^{\prime}(50)\end{array}$ & 1.113 & 1.113 & $\begin{array}{l}01(34) \\
0(35)\end{array}$ & 1.98 & & $\begin{array}{l}\text { W(21) } \\
Q(25)\end{array}$ & 1.265 & 1452 \\
\hline $\begin{array}{l}q(j)- \\
\text { His }\end{array}$ & 1.113 & 1.113 & $\begin{array}{c}\cos _{1}(1) \\
Q_{1}\end{array}$ & 1.994 & 1.45 & $\begin{array}{l}G(9)- \\
\mathbb{N}(24)\end{array}$ & 1.256 & 1452 \\
\hline $\begin{array}{l}C(37) \\
\text { Hisi) }\end{array}$ & 1.113 & 1.113 & $\begin{array}{l}\sigma^{3} 35 \\
\cos ^{\prime} 17\end{array}$ & 1.55 & 1.45 & $\begin{array}{l}\text { c18) } \\
\text { (42) }\end{array}$ & 1.497 & 1497 \\
\hline $\begin{array}{l}C(1) \\
\mathrm{H}(5)\end{array}$ & 1.1 & 1.1 & $\begin{array}{l}N(7) \\
\cos 17\end{array}$ & 1.56 & & $\begin{array}{l}010 \% \\
\mathbb{N}(21)\end{array}$ & 1.265 & 1452 \\
\hline $\begin{array}{l}030 \\
\mathrm{H} 59\end{array}$ & 1.1 & 1.1 & $\begin{array}{c}\cos (17) \\
\mathbb{N} 24\end{array}$ & 1.50 & & $\begin{array}{l}\text { Why- } \\
\text { Qn }\end{array}$ & 1256 & 1452 \\
\hline $\begin{array}{l}729 \\
H^{\prime} 50\end{array}$ & 1.1 & 11 & $\begin{array}{l}(10) \\
0(35)\end{array}$ & 1355 & 1.353 & $\begin{array}{l}\text { Whr } \\
\mathbb{N}(22)\end{array}$ & 1095 & \\
\hline $\begin{array}{l}\text { (12) } \\
\text { H57 }\end{array}$ & 1.1 & 11 & $\begin{array}{l}\mathrm{N}(\mathrm{T}) \\
0(3)\end{array}$ & $1.4 i$ & $1.4 i$ & $\begin{array}{l}\mathrm{Q}(9) \\
Q(20)\end{array}$ & 1357 & 58 \\
\hline $\begin{array}{l}077 \\
\mathrm{H}^{5}(53)\end{array}$ & 1.113 & 1.113 & $\begin{array}{l}0(1)- \\
g(25)\end{array}$ & 1357 & 1.42 & $\begin{array}{l}018- \\
019)\end{array}$ & 1957 & 1337 \\
\hline $\begin{array}{l}920 \\
0(33)\end{array}$ & 1.535 & 1.335 & $\begin{array}{l}\gamma(9) \\
\sigma(31)\end{array}$ & 1337 & 1.42 & $\begin{array}{l}\text { (5) } \\
\alpha 15)\end{array}$ & 1355 & 1335 \\
\hline $\begin{array}{r}N(27) \\
(72)\end{array}$ & 1.47 & 1.47 & $\begin{array}{l}929 \\
200\end{array}$ & 1377 & 1.42 & $\begin{array}{l}N(7) \\
Q 15)\end{array}$ & 1.47 & 147 \\
\hline
\end{tabular}


Farah M. Ibrahim

Table(8)

Selected molecular structure parameters (Bond angle) of CoMAP.

\begin{tabular}{|c|c|c|c|c|c|c|c|c|}
\hline Atorms: & Actual & gptimal & Atoms & Actual & optimal & Atoms & Antual & optimal \\
\hline $\begin{array}{l}\mathrm{C}(30)- \\
\mathrm{H}(59)\end{array}$ & $1 \_1$ & 11 & $\begin{array}{l}\operatorname{cy}(34)- \\
\operatorname{Cl}(35)\end{array}$ & 198 & & $\begin{array}{l}\text { N(24)- } \\
C(19)- \\
C(20)\end{array}$ & 111 & 120 \\
\hline $\begin{array}{l}\mathrm{C}(2 \mathrm{~g})- \\
\mathrm{H}(5 \mathrm{~B})\end{array}$ & $1 \_1$ & 11 & $\begin{array}{c}\operatorname{co}(17)- \\
\alpha(16)\end{array}$ & 1.7954 & 145 & $\begin{array}{l}\text { W(24)- } \\
C(19)- \\
c(18)\end{array}$ & 120 & 120 \\
\hline $\begin{array}{l}\mathrm{C}(28)- \\
\mathrm{H}(57)\end{array}$ & 1.1 & II & $\begin{array}{l}\alpha(35)- \\
\cos (17)\end{array}$ & 1.8596 & 145 & $\begin{array}{l}c(20)- \\
c(19)- \\
c(18)\end{array}$ & 111 & 120 \\
\hline $\begin{array}{l}\mathrm{O}(31)- \\
\mathrm{H}(60)\end{array}$ & $1 \ldots 1$ & 11 & $\begin{array}{l}\mathrm{N}(7)- \\
\operatorname{co}(17)\end{array}$ & 1.836 & & $\begin{array}{l}C(23)- \\
C(18)- \\
N(22)\end{array}$ & 1245 & 1253 \\
\hline $\begin{array}{l}\mathrm{Cl}(34)- \\
\mathrm{Cl}(35)\end{array}$ & 198 & & $\begin{array}{l}\cos (17)- \\
N(24)\end{array}$ & 1.836 & & $\begin{array}{l}C(23)- \\
c(18)- \\
c(19)\end{array}$ & 1245 & 1214 \\
\hline $\begin{array}{c}\operatorname{Co}(17)- \\
O(15)\end{array}$ & 1.7954 & 145 & $\begin{array}{l}C(20)- \\
\alpha(33)- \\
C o(17)\end{array}$ & 98:5759 & & $\begin{array}{l}N(22)- \\
C(18)- \\
C(19)\end{array}$ & 111 & 120 \\
\hline $\begin{array}{l}O(33)- \\
C o(17)\end{array}$ & 1.8595 & 145 & $\begin{array}{l}\text { H(O3)- } \\
C(32)- \\
H(62)\end{array}$ & 10952 & 109 & $\begin{array}{l}\text { O(33)- } \\
\text { Co(17)- } \\
\text { N(24) }\end{array}$ & 832186 & \\
\hline $\begin{array}{l}N(7)- \\
C \alpha(17)\end{array}$ & 1.836 & & $\begin{array}{l}H(63)- \\
C(32)- \\
H(61)\end{array}$ & 10944618 & 109 & $\begin{array}{l}\text { O(33)- } \\
\mathrm{Co}(17)- \\
\mathrm{O}(16)\end{array}$ & 1069214 & \\
\hline $\begin{array}{l}\operatorname{Co}(17)- \\
N(2-4)\end{array}$ & 1.835 & & $\begin{array}{l}H(03)- \\
C(32)- \\
N(22)\end{array}$ & 1094618 & & $\begin{array}{l}\text { O(j3)- } \\
\text { Co(17)- } \\
\text { in(7) }\end{array}$ & 1590124 & \\
\hline
\end{tabular}<smiles></smiles><smiles>O=S(=O)([O-])[O-]</smiles>

Chemical Formula: $\mathrm{C}_{24} \mathrm{H}_{30} \mathrm{~N}_{6} \mathrm{NiO}_{6} \mathrm{~S}$

Exact Mass: 588.13

Molecular Weight: 589.29

m/z: 588.13 (100.0\%), $590.13(45.1 \%), 589.13(29.2 \%), 591.13(13.2 \%), 592.12(7.1 \%), 590.14$

(3.4\%), $592.13(2.8 \%), 593.13(1.7 \%), 594.12(1.7 \%), 591.12(1.3 \%)$
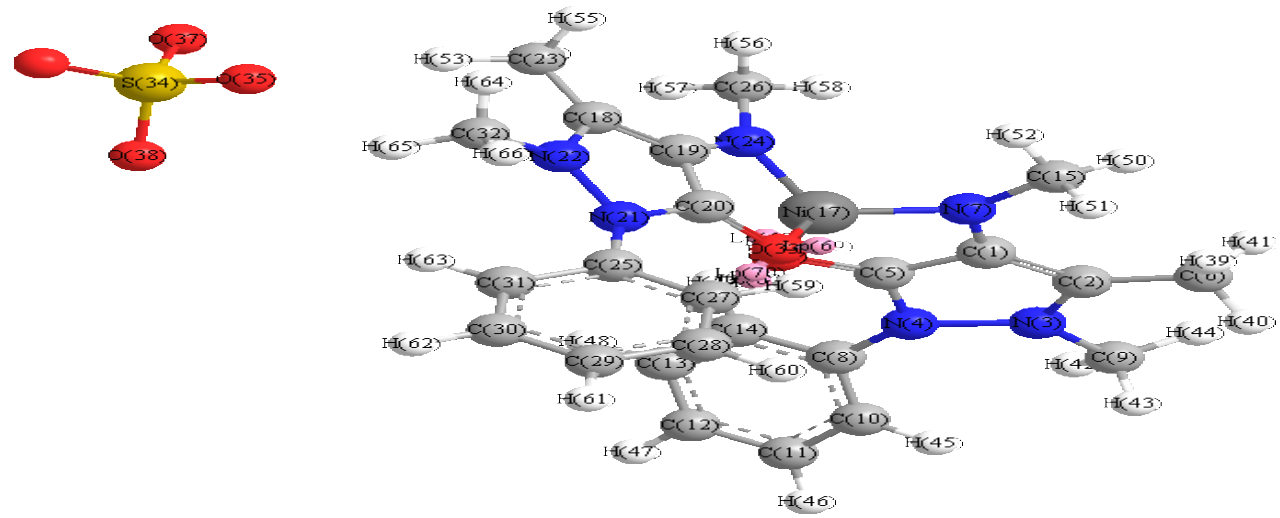

Fig.(10): Serial number of atoms of NiMAP. 
Table(9)

Selected molecular structure parameters of Bond lengths $(\AA)$ for NiMAP.

\begin{tabular}{|c|c|c|c|c|c|c|c|c|}
\hline Atrous & Actual & optimial & Attoms & Actual & qutimilal & stoms & Actulal & quimal \\
\hline $\begin{array}{l}\alpha(97) \\
\mathrm{L} p 70\end{array}$ & 056 & 06 & $\begin{array}{l}9(9) \\
\mathrm{H}(4)\end{array}$ & 1.113 & 1.113 & $\begin{array}{l}\text { (19) } \\
\text { (124) }\end{array}$ & 1.386 & 1462 \\
\hline $\begin{array}{l}\alpha(3) \\
\mathrm{Lip}(6)\end{array}$ & 0.001 & 06 & $\begin{array}{l}99 \\
\mathrm{H}(43)\end{array}$ & 1.1131 & 1.113 & $\begin{array}{l}O(18) \\
(12)\end{array}$ & 15014 & 1497 \\
\hline $\begin{array}{l}\overline{10} \\
\mathrm{~L} / 60\end{array}$ & 0.002 & 0.6 & $\begin{array}{c}\text { (9) } \\
\mathrm{H} 4 \mathrm{I})\end{array}$ & 1.1122 & 1.115 & $\begin{array}{l}Q(20) \\
\text { (1) }\end{array}$ & 1356 & $14 \sqrt{2}$ \\
\hline $\begin{array}{l}\alpha 16 \\
\mathrm{~L} 0607\end{array}$ & 0,566 & 0.6 & $\begin{array}{r}\text { Ni17) } \\
\text { (19) }\end{array}$ & 1.745 & & $\begin{array}{l}\mathrm{N}(21) \\
(12)\end{array}$ & 1.370 & 140 \\
\hline $\begin{array}{l}637 \\
H 66)\end{array}$ & 1.112 & 1.113 & $\alpha(3)$ & 1.745 & & $\begin{array}{l}\text { N(1) } \\
\text { M(2) }\end{array}$ & 1.445 & \\
\hline $\begin{array}{l}6 / 5 \\
H(5)\end{array}$ & |.1131 & 1.15 & $\begin{array}{r}\mathbb{N i} \\
\mathrm{M}(17)\end{array}$ & 1.76 & & $\begin{array}{l}\mathrm{O}(9) \\
(10)\end{array}$ & 1.417 & $10^{2}$ \\
\hline $\mathrm{N}(\mathrm{p}) \mathrm{Q}$ & $1.4 L^{5}$ & 1.47 & $\begin{array}{l}\mathrm{N}(17) \\
\mathrm{M}(4)\end{array}$ & 1.56 & & $\begin{array}{l}q(8) \\
(19)\end{array}$ & $1.4 W 4$ & 133 \\
\hline 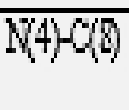 & 1.477 & 1.402 & $(\phi) \propto(1)$ & 1.4175 & 150 & $\begin{array}{l}\phi 5 \\
\alpha 16)\end{array}$ & 1.3605 & 135 \\
\hline $\begin{array}{l}N(7) \\
015\end{array}$ & 1.448 & 1.47 & $\mathrm{~N} 440$ & 1.357 & 1.462 & $\frac{\partial 27}{\partial 2)}$ & 1.342 & 142 \\
\hline $\begin{array}{r}014 \\
\phi 0)\end{array}$ & 1.4357 & 1.42 & $(\mathrm{O}) \mathrm{N}(\mathrm{S})$ & 1.3706 & 1.46 & $\begin{array}{l}q(5) \\
(27)\end{array}$ & $1.40 \%$ & $\overline{142}$ \\
\hline
\end{tabular}

Table (10)

Selected molecular structure parameters (Bond angle) of NiMAP.

\begin{tabular}{|c|c|c|c|c|c|c|c|c|}
\hline $\begin{array}{l}\alpha 38- \\
s(34)- \\
\alpha 37)\end{array}$ & 108.0853 & & $\begin{array}{l}c(31) \\
q(25) \\
\alpha(27)\end{array}$ & 118.5985 & 120 & $\begin{array}{l}c(2)- \\
c 19- \\
(18)\end{array}$ & 1112147 & 120 \\
\hline $\begin{array}{l}\alpha 38)- \\
\$(34)- \\
\alpha 36)\end{array}$ & 108.185 & & $\begin{array}{l}(31)- \\
(25)- \\
N(21)\end{array}$ & 1199405 & 120 & $\begin{array}{l}\text { (25)- } \\
\text { (18)- } \\
\text { N(22) }\end{array}$ & 1286899 & 1253 \\
\hline $\begin{array}{l}\alpha(38) \\
\$(34)- \\
\alpha(35)\end{array}$ & 108.135 & & $\begin{array}{l}9(27)- \\
\alpha(25) \\
\text { (21) }\end{array}$ & 121.4204 & 120 & $\begin{array}{l}\text { (25) } \\
\text { (18) } \\
\text { (19) }\end{array}$ & 1269047 & 1214 \\
\hline $\begin{array}{l}\alpha 36)- \\
\$(34) \\
\alpha 35)\end{array}$ & 115.8429 & 1166 & $\begin{array}{l}\text { (25)- } \\
\text { N(24) } \\
C(19)\end{array}$ & 153385 & 108 & $\begin{array}{l}\text { (22)- } \\
\text { (18)- } \\
\text { (19) }\end{array}$ & 1057585 & 120 \\
\hline $\begin{array}{l}\operatorname{Lg}(70) \\
\alpha(33)- \\
\operatorname{Lg}(5)\end{array}$ & $125.4 \Omega 26$ & 131 & $\begin{array}{l}\text { (26) } \\
\text { N(24) } \\
\mathrm{N}(17)\end{array}$ & 130.7335 & & $\begin{array}{l}\alpha(33)- \\
\mathrm{N}(17)- \\
\mathrm{N}(24)\end{array}$ & 99.7292 & \\
\hline $\begin{array}{l}L(0)- \\
\alpha(33)- \\
c(20)\end{array}$ & 105.4315 & 10326 & $\begin{array}{l}\text { Q19) } \\
\text { N(24) } \\
\text { N(17) }\end{array}$ & 103.7237 & 109 & $\begin{array}{l}\alpha(3)- \\
\bar{N}(17)- \\
\alpha 10)\end{array}$ & 1117717 & \\
\hline $\begin{array}{l}C(32)- \\
H(64)\end{array}$ & 1.1126 & 1113 & $\begin{array}{l}\text { H(55) } \\
\text { (25)- } \\
\text { H54) }\end{array}$ & 1089485 & 109 & $\begin{array}{l}\alpha 33)- \\
\mathrm{N}(17)- \\
\mathrm{N}(\mathrm{l})\end{array}$ & 1135786 & \\
\hline $\begin{array}{l}C(31)- \\
\left.H^{\prime} 63\right)\end{array}$ & 1.1013 & 11 & $\begin{array}{l}\alpha(3)- \\
(20)- \\
\alpha(19)\end{array}$ & 1215966 & 124.3 & $\begin{array}{l}\text { N(4)- } \\
N(3)- \\
C(2)\end{array}$ & 1109255 & \\
\hline $\begin{array}{l}C(30)- \\
\mathrm{H}(62)\end{array}$ & 1.1022 & 11 & $\begin{array}{l}\text { N(21)- } \\
\text { (20)- } \\
(19)\end{array}$ & 108.1381 & 120 & $\begin{array}{l}\text { N( })- \\
c(5) \\
(5)\end{array}$ & 1133523 & 120 \\
\hline $\begin{array}{l}C(2)- \\
Y(61)\end{array}$ & 1.102 & 11 & $\begin{array}{l}\text { N(24) } \\
\text { (19)- } \\
c(20)\end{array}$ & 113.3058 & 120 & $\begin{array}{l}\text { N(7)- } \\
\text { (1)- } \\
c(2)\end{array}$ & 1352701 & 120 \\
\hline
\end{tabular}


Farah M. Ibrahim
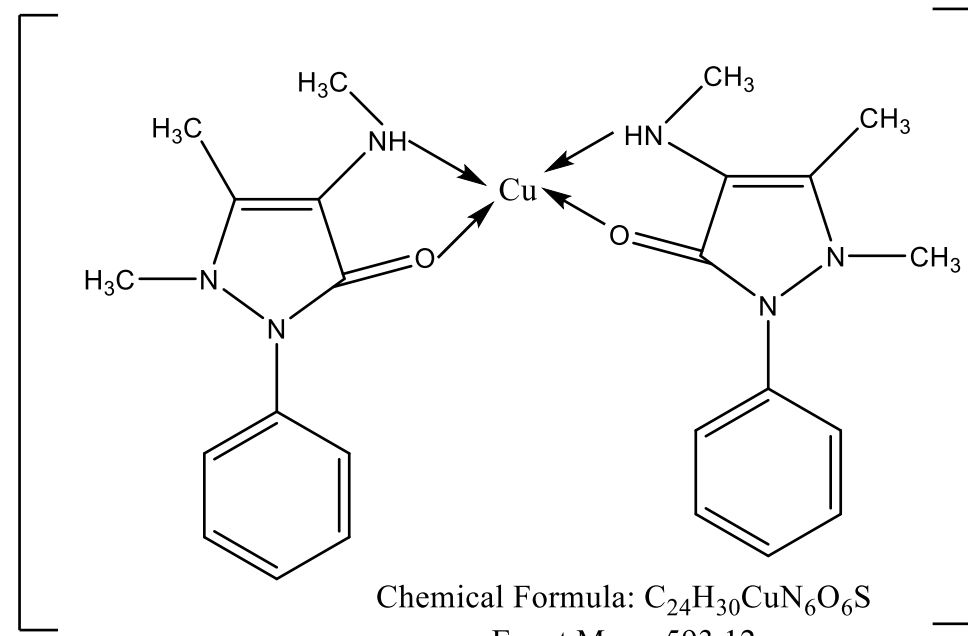<smiles>O=S(=O)([O-])[O-]</smiles>

Exact Mass: 593.12

Molecular Weight: 594.14

m/z: 593.12 (100.0\%), 595.12 (49.7\%), 594.13 (26.5\%), 596.13 (12.6\%), $595.13(4.8 \%), 594.12$

(3.0\%), $596.12(2.6 \%), 597.12(2.4 \%), 597.13(2.3 \%)$

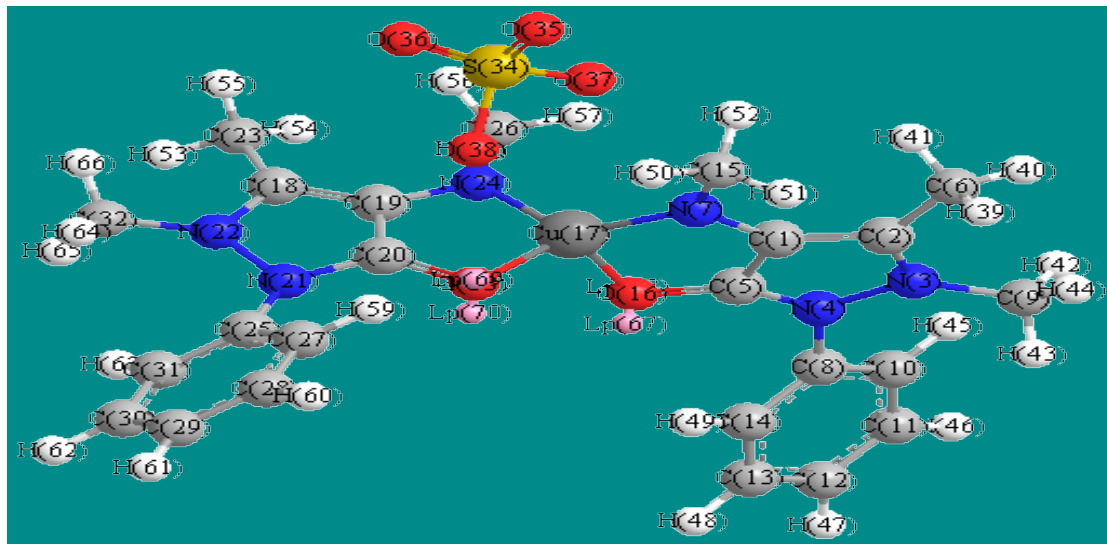

Fig.(11): Serial number of atoms of CuMAP.

Table (11)

Selected molecular structure parameters (Bond lengths, $\AA$ ) of CuMAP

\begin{tabular}{|c|c|c|c|c|c|c|c|c|}
\hline Atams & Actual & optimal & Atoms & Actual & gotimal & Atoms & Actual & optimal \\
\hline $\begin{array}{l}\alpha(33) \\
\operatorname{Ig}(0)\end{array}$ & 0.5972 & 0.6 & $C(5)-C(1)$ & 14122 & 1502 & $C(13)-C(14)$ & 13945 & 1.42 \\
\hline $\begin{array}{l}\alpha(35) \\
\operatorname{Lg}(\Theta)\end{array}$ & 0.6001 & 0.6 & $\bar{N}(4)-C(5)$ & 13559 & $14 \sqrt{2}$ & $C(12)-C(13)$ & 1396 & 1.42 \\
\hline $\begin{array}{l}\text { Q16) } \\
\text { Lo( })\end{array}$ & 0.6005 & 0.6 & $C(2)-\mathrm{N}(3)$ & 13722 & $14 \sqrt{2}$ & $C(11)-C(12)$ & 13987 & 1.42 \\
\hline $\begin{array}{l}\alpha(16) \\
\text { Lo(6i) }\end{array}$ & 0.5988 & 0.6 & $\begin{array}{l}C(27)- \\
\left.H^{5} 59\right)\end{array}$ & 11005 & 11 & $C(10)-C(11)$ & 13933 & 1.42 \\
\hline $\begin{array}{l}\$(34) \\
0(36)\end{array}$ & 1.4496 & 1.45 & $\begin{array}{l}C(25) \\
\left.H^{5} 58\right)\end{array}$ & 11128 & 1113 & $c(8)-C(10)$ & 14062 & 1.42 \\
\hline $\begin{array}{l}\text { S(34) } \\
0(35)\end{array}$ & 1.449 & 1.45 & $\begin{array}{l}C(25) \\
H^{5} 57\end{array}$ & 11127 & 115 & $\mathrm{~N}(3)-(9)$ & 14714 & 1.47 \\
\hline $\begin{array}{l}\text { Q10)- } \\
\text { cur(17) }\end{array}$ & 1.297 & & $\begin{array}{l}\text { Q(15)- } \\
\left.H^{5} 56\right)\end{array}$ & 11125 & 1113 & $\mathrm{~N}(4)-\mathrm{C}(8)$ & 14415 & 1.452 \\
\hline $\begin{array}{l}\text { N(7)- } \\
\text { cu(1) }\end{array}$ & 1.8241 & & $\begin{array}{l}C(25) \\
\left.H^{5} 55\right)\end{array}$ & 11134 & 1113 & $C(1)-\mathrm{N}(7)$ & 13867 & 1.452 \\
\hline $\begin{array}{l}\text { N(24) } \\
\text { cu(1) }\end{array}$ & 1.8242 & & $\begin{array}{l}C(25) \\
\left.H^{5} 54\right)\end{array}$ & 11093 & 1113 & $C(2)-C(6)$ & 1501 & 1.497 \\
\hline $\begin{array}{l}\alpha(3)- \\
c 4(17)\end{array}$ & 1.8107 & & $\begin{array}{l}C(23)- \\
\left.H^{\prime} 53\right)\end{array}$ & 1113 & 1113 & $N(22)-C(32)$ & 14721 & 1.47 \\
\hline
\end{tabular}


Table (12)

Selected molecular structure parameters (Bond angle) of CUMAP.

\begin{tabular}{|c|c|c|c|c|c|c|c|c|}
\hline $\begin{array}{l}\text { Attoms } \\
\text { O(36)- } \\
\text { s(34) } \\
0(35)\end{array}$ & $\begin{array}{r}\text { Actual } \\
1184972\end{array}$ & $\begin{array}{l}\text { optimal } \\
1166\end{array}$ & $\begin{array}{l}\text { Atoms } \\
N(21)- \\
c(20)- \\
C(19)\end{array}$ & $\begin{array}{r}\text { Actual } \\
1085 \% 02\end{array}$ & Gptimal & $\begin{aligned} & \text { Atcoms } \\
& \text { N(24)-Cu(17)- } \\
&\propto 16)\end{aligned}$ & $\begin{array}{r}\text { Actual } \\
1055545\end{array}$ & optimal \\
\hline 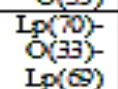 & 118.3634 & 131 & $\begin{array}{c}\text { N(24)- } \\
(19)- \\
C(20)\end{array}$ & 1127167 & 120 & $\begin{array}{r}\mathrm{N}(24)-\mathrm{Cu}(17)- \\
\mathrm{N}(7)\end{array}$ & 1305612 & \\
\hline $\begin{array}{l}\text { Lp(N)- } \\
\alpha(33)- \\
C(20)\end{array}$ & 1008591 & 10326 & $\begin{array}{l}N(24)- \\
C(19)- \\
C(18)\end{array}$ & 1563091 & 120 & 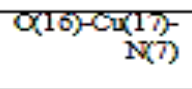 & 931362 & \\
\hline $\begin{array}{c}\mathrm{H}(\mathrm{Bg})- \\
C(31) \\
C(30)\end{array}$ & 119.0224 & 120 & $\begin{array}{l}C(20)- \\
\stackrel{C(19)-}{C(18)}\end{array}$ & 1109709 & 120 & $\begin{array}{r}L_{p}(\infty)-\alpha(16)- \\
L p(67)\end{array}$ & 121.0831 & 131 \\
\hline $\begin{array}{l}\text { H(d) } \\
\text { C(31) } \\
C(25)\end{array}$ & 1201667 & 120 & $\begin{array}{l}\text { (2) } \\
\text { O(18)- } \\
\text { N(2) }\end{array}$ & 128.3828 & 1253 & 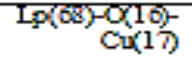 & 1163027 & \\
\hline $\begin{array}{l}\mathrm{H}(\mathrm{s})- \\
\mathrm{C}(31)- \\
(\mathrm{C})\end{array}$ & 119.0224 & 120 & $\begin{array}{l}\mathrm{H}(45)- \\
(10)-\end{array}$ & 1192016 & 120 & $\frac{1 p(\sigma)-\alpha(16)}{C(S)}$ & 1039855 & 10326 \\
\hline $\begin{array}{l}\text { C(26)- } \\
N(24)- \\
C U(1)\end{array}$ & 1306428 & & $\begin{array}{r}H(45) \\
C(10)-C(8)\end{array}$ & 1201486 & 120 & $\begin{array}{r}N_{(24)-C(17)-} \\
\propto(16)\end{array}$ & $15 \$ 545$ & \\
\hline $\begin{array}{l}C(9)- \\
N(24)- \\
C u(17)\end{array}$ & 105.8222 & & $\begin{array}{l}\mathrm{H}(45)- \\
\mathrm{C}(10)- \\
\mathrm{C(11)}\end{array}$ & 1192016 & 120 & $\mathrm{Cu}(17)-\alpha(16)$ & 103.7855 & \\
\hline
\end{tabular}

\section{Conclusion}

The theoretical study of Hyperchem 8.0.7 program using Semi-empirical calculations and PM3 method help to characterized MAP complexes by calculate optimized geometries, HOMO, LUMO, electrostatic potential and vibrational frequencies, these data are shown good agreement with the experimental data that which were used elemental and spectroscopic analysis. Chemoffice program used to draw the structure of MAP complexes and calculate the CHNOS analysis, also Chemoffice 3D used to draw 3D structure of molecules, bond distances and bond angles.

\section{Reference}

[1] Ergun H., Frattarelli D.A.C., Aranda J.V.J., "Characterization of the role of physicochemical factors on the hydrolysis of dipyrone", J. Pharm. Biomed. Anal.,35, 479-487, 2004.

[2] Mario G., Virginia D. V., Hong K. L., Fulvio L., Cezary K.,Vanessa F., Artur B., Cecilia V., "Pharmacokinetic investigations of the marker active metabolite-4methylamino-antipyrin after intravenous and intramuscular injection of metamizole in healthy sheep", 132, 143-146, 2015.

[3]Sigroha S., Narasimhan B., Kumar P., Khatkar A., Ramasamy K., Mani V., Mishra R. K., Abdul Majeed AB., "Design, Synthesis, Antimicrobial, Anticancer Evaluation, and QSAR studies of 4(substitutedbenzylide-amino)-1,5dimethyl-2-phenyl-1,2-dihydropyrazol-3- ones", Med. Chem. Res., 21(11), 38633875, 2012.

[4] Teng Y., Liu R., Li C., Zhang H., "Effect of 4-aminoantipyrine on Oxidative Stress Induced by Glutathione Depletion in single Human Erythrocytes using a Micro Fluidic device together with Fluorescence Imaging”, J Hazardous Materials, 192, 1766-1771, 2011.

[5] Joseph J., Nagashri K., Ayisha Bibin Rani G., "Synthesis, characterization and antimicrobial activities of copper complexes derived from 4-aminoantipyrine derivatives", Journal of Saudi Chemical Society, 17(3), 285-294, 2013.

[6] Salem O., Thabet H., Ashraf F. and Fathiya A., "Study on the relationship between genetic polymorphisms of cytochrome $\mathrm{P} 450$ and effects of Methylaminoantipyrine in dental pain", Journal of Chemical and Pharmaceutical Research, 8(4),1365-1373, 2016.

[7] Rezende R. M., França D. S., Menezes G. B., dos Reis W. G. P., Bakhle Y. S. and Francischi J. N., "Different mechanisms underlie the analgesic actions of paracetamol and dipyrone in a rat model of inflammatory pain”, $\mathrm{Br} \mathrm{J}$ Pharmacol., 153(4),760-768, 2008.

[8] Yi L., Yuanyuan L., Haowei W., Xiaohui X., Ping W. and Fangshi L., "Synthesis, Crystal Structure, Vibration Spectral, and DFT Studies of 4-Aminoantipyrine and Its Derivatives", Molecules, 18, 877-893, 2013. 
[9] Mostafa M. G., Marwa G. and Mansour S., "Synthesis, Characterization and AntiBreast Cancer Activity of New 4Aminoantipyrine-Based Heterocycles", Int. J. Mol. Sci., 15, 7539-7553, 2014.

[10] Abdullah M. and Salman A., "Synthesis and Anti-Bacterial Activities of Some Novel Schiff Bases Derived from Aminophenazone", Molecules, 15, 68506858, 2010.

[11] Priti D., Pradeep K. S., Amit K., Anand K. H., Renu D., "4-Aminoantipyrine: A Significant Tool for the Synthesis of Biologically Active Schiff Bases and Metal Complexes", Int. J. Pharm. Sci. Rev. Res., 34(1), 162-170, 2015.

[12] Thamarai S., Mahalakshmi S., "Synthesis and Characterization of new Heterocyclic Schiff base ligand derived from 4-Amino Antipyrine", International Journal of Advance Research and Development, 2(2), 51-56, 2017.

[13] Acha U. E., Olalere G. A., Opeyemi A. A., Nelson O., "Computational and Experimental Studies of 4-Aminoantipyrine as Corrosion Inhibitor for Mild Steel in Sulphuric Acid Solution", Int. J. Electro chem. Sci., 7, 534-553, 2012 .

[14] Robert M.S., Francis X.W., David J. K., David L.B., Spectrometric Identification of Organic Compounds, John Wiely and Sons, edition $(8)^{\text {th }}, 2015$.

[15] Rajasekar K. and Ramachandramoorthy T., "Synthesis, Spectral characterization and biological activities of $\mathrm{Cr}$ (III), $\mathrm{Co}(\mathrm{II}), \mathrm{Ni}(\mathrm{II})$ and $\mathrm{Cd}(\mathrm{II})$ complexes with 4aminoantipyrineand thiocyanate ion as ligands", Int J Pharm Bio Sci, 4(2), 271 276, 2013.

[16] Tudor R., Simona P., Veronica L., Carmen Ch. and Raluca C., "Copper (II) Complexes with Ligands Derived from 4Amino-2,3-dimethyl-1-phenyl-3-pyrazolin5-one: Synthesis and Biological Activity", Molecules, 11, 904-914, 2006.

[17] Aayad A.S., Hussain J.M., "Thermal Analysis of Different Pyrazolone Azo Derivative and Their Complexes with $\mathrm{Pd}(\mathrm{II}), \mathrm{Ni}(\mathrm{II})$ and $\mathrm{Ag}(\mathrm{I})$ ", International Journal of Science and Research (IJSR), 4 (6), 504-508, 2015. 